\title{
Quantum Cellular Automata, Tensor Networks, and Area Laws
}

\author{
Lorenzo Piroli $\oplus^{1,2}$ and J. Ignacio Cirac ${ }^{1,2}$ \\ ${ }^{1}$ Max-Planck-Institut für Quantenoptik, Hans-Kopfermann-Straße 1, 85748 Garching, Germany \\ ${ }^{2}$ Munich Center for Quantum Science and Technology, Schellingstraße 4, 80799 München, Germany
}

(Received 7 August 2020; accepted 8 October 2020; published 2 November 2020)

\begin{abstract}
Quantum cellular automata are unitary maps that preserve locality and respect causality. We identify them, in any dimension, with simple tensor networks (projected entangled pair unitary) whose bond dimension does not grow with the system size. As a result, they satisfy an area law for the entanglement entropy they can create. We define other classes of nonunitary maps, the so-called quantum channels, that either respect causality or preserve locality. We show that, whereas the latter obey an area law for the number of quantum correlations they can create, as measured by the quantum mutual information, the former may violate it. We also show that neither of them can be expressed as tensor networks with a bond dimension that is independent of the system size.
\end{abstract}

DOI: 10.1103/PhysRevLett.125.190402

Causality is a fundamental concept in physics. It states that physical actions cannot propagate in space at an arbitrary speed. In quantum physics, this can be mathematically captured by the notion of quantum cellular automata (QCA) [1,2]. These are the most general unitary maps between quantum states that act in discrete space (i.e., in lattices) and time and respect causality [3-6]. They can be viewed as the quantum version of classical cellular automata, which are systems with discrete variables evolving under a local update rule. In the past years, a great deal of progress has been made in the characterization of QCA. So far, complete solutions have been obtained in one [7] and two spatial dimensions [8-11]. Additionally, in the first case, QCA have been identified [12-18] with matrix product operators, a $1 D$ version of tensor networks (TN), which satisfy an extra condition named simpleness [14] (this has been recently extended to fermionic systems $[19,20])$. This identification connects QCA with TN, a very active area of research in many-body physics and quantum information. While most of the progress on QCA has been on unitary maps, very little is known about quantum channels representing more general physical actions [3,4,21], for which it is not even clear how to properly define them.

In this work, we investigate the connections between QCA and TN $[22,23]$ and characterize them in terms of the amount of entanglement and correlations they can create. First, we identify QCA in any dimension as projected

Published by the American Physical Society under the terms of the Creative Commons Attribution 4.0 International license. Further distribution of this work must maintain attribution to the author(s) and the published article's title, journal citation, and DOI. Open access publication funded by the Max Planck Society. entangled pair unitary (PEPU) operators that are also simple and with a bond dimension that does not grow with the lattice size. We also show that the amount of entanglement generated by the action of a QCA is limited by an area law, similar to the one that characterizes the ground states of local Hamiltonians [24]. Additionally, we analyze two natural extensions of nonunitary QCA: causality-preserving quantum channels (CPQC) and locality-preserving quantum channels (LPQC). While the former satisfies causality, the latter cannot create longrange correlations and fulfills an area law for the quantum mutual information. The LPQC are a strict subset of CPQC, and, unlike QCA, they cannot be expressed as TN with a fixed bond dimension.

$Q C A$ and quantum channels.-We consider $N=M^{d_{L}}$ qudits in a finite regular lattice in $d_{L}$ dimensions. The lattice is characterized by a graph $\mathcal{G}=(V, E)$, where the qudits are at the vertices $V$, which are represented by a vector $n \in \mathbb{Z}^{d_{L}}$, and the edges $e_{n, m} \in E$ if $|n-m|=1$ for open boundary conditions, and similarly for periodic boundary conditions. The coordination number is $z=2 d_{L}$. The edges define a metric: The distance between the vertices, $\delta(n, m)$, is the minimum number of edges that connects them. The Hilbert space associated with the set of qudits is $\mathcal{H}=\otimes_{n \in V} \mathcal{H}_{n}$, where $\operatorname{dim}\left(\mathcal{H}_{n}\right)=d$ is the physical dimension. For $r \leq M / 4$ and a subset $A \subset V$, we define its $r$ neighborhood: $a_{r}=\{n \in V \backslash A: \delta(n, A) \leq r\}$, and $\bar{A}_{r}=A \cup a_{r}$. We further define the $r$-next-nearest neighborhood: $b_{r}=a_{2 r} \backslash a_{r}$, and $B_{r}=V \backslash\left(\bar{A}_{r} \cup b_{r}\right)$, so that $\bar{B}_{r}=B_{r} \cup b_{r}$ is the complement of $\bar{A}_{r}$ (see Fig. 1). We denote by $S$ all the sets $A$ such that $B_{r}$ is not empty. For a given $A \in S$ and $r$, the Hilbert space is decomposed as

$$
\mathcal{H}=\mathcal{H}_{A} \otimes \mathcal{H}_{a_{r}} \otimes \mathcal{H}_{b_{r}} \otimes \mathcal{H}_{B_{r}}=\mathcal{H}_{\bar{A}_{r}} \otimes \mathcal{H}_{\bar{B}_{r}}
$$


(a)

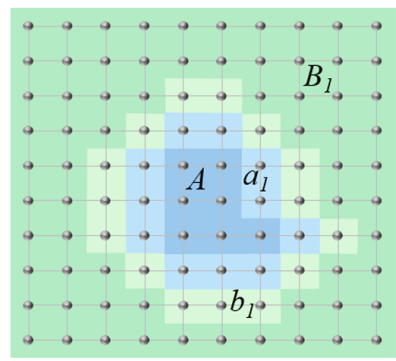

(b)

(c)
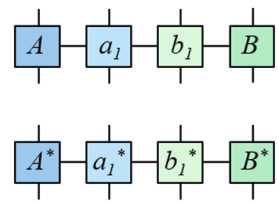

(d)

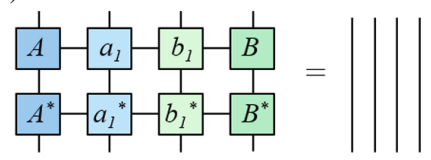

FIG. 1. (a) Example of the different subsets defined in the text for $r=1$ : $A$ is in dark blue and its neighborhood $a_{1}$ in light blue. Their union is $\bar{A}_{1} . B_{1}$ and $b_{1}$ are in dark and light green, respectively, and their union is $\bar{B}_{1}$. (b)-(d) PEPU representation of $U, U^{\dagger}$, and graphical notation for the identity $U U^{\dagger}=\mathbb{1}$, respectively.

Finally, for $C \subset V$, we denote by $\operatorname{tr}_{C}$ the trace in $\mathcal{H}_{C}$ and by $X_{C}$ an operator supported on that space.

Let us now introduce a special type of quantum channels (QC) $\mathcal{E}$ acting on the qudits, i.e., trace-preserving completely positive maps [25]. We will denote by $\mathcal{E}^{\dagger}$ their adjoints with respect to the Hilbert-Schmidt inner product, describing the action in the Heisenberg picture. Then

Definition 1: A CPQC on the lattice $\mathcal{G}$ with range $r$ is a channel $\mathcal{E}$ such that, for any $A \in S$ and $X_{A}$, there exists some $X_{\bar{A}_{r}}$ such that $\mathcal{E}^{\dagger}\left(X_{A}\right)=X_{\bar{A}_{r}}$.

Our definition is equivalent to that presented in Ref. [2]. It states that, for an observable localized at site $x$, the expectation value on the evolved state is determined by the restriction of the initial state on a neighborhood of $x$, thus justifying the name causality preserving. When $\mathcal{E}$ is defined by a unitary operator $U$, namely, $\mathcal{E}(X)=U X U^{\dagger}$ for all $X \in L(\mathcal{H})$, the set of linear operators acting on $\mathcal{H}$, we will say that the QC is unitary. Then, QCA are simply unitary CPQC. In such a case, $\mathcal{E}^{\dagger}(X)=U^{\dagger} X U$, and $\mathcal{E}^{\dagger}$ is still a QCA with the same range as $\mathcal{E}$ [8].

Before proceeding, let us mention that we could have considered more general graphs $\mathcal{G}$, as long as they have no double edges nor self-loops. This would include other lattice geometries or topologies, but it would make the notation more cumbersome. Thus, in the following, we will set $r=1$, drop the corresponding subindex in the sets $a, b$, and $B$, and take $M \geq 4$ [26].

We introduce now another class of QC:

Definition 2. $\mathcal{E}$ is a LPQC if, for any $A \in S$ and $\rho_{\bar{A}, \bar{B}} \geq 0$,

$$
\operatorname{tr}_{a, b}\left[\mathcal{E}\left(\rho_{\bar{A}} \rho_{\bar{B}}\right)\right]=\frac{1}{d^{N}} \operatorname{tr}_{a, \bar{B}}\left[\mathcal{E}\left(\rho_{\bar{A}}\right)\right] \operatorname{tr}_{\bar{A}, b}\left[\mathcal{E}\left(\rho_{\bar{B}}\right)\right]
$$

This means that, if we act on a product state with the quantum channel, no correlation is created between the regions $A$ and $B$. Intuitively, this corresponds to a form of localization in the Schrödinger picture, which, as we will see, represents a stronger condition than causality preservation.

Choi-Jamiolkowski state.-Instead of dealing with channels, it will be useful to work with the corresponding Choi-Jamiolkowski states (CJS) [28]. We associate an extra ancilla with each qudit, so that we get a copy of the lattice with vertices $V^{\prime}$. We also take $\Phi=|\Phi\rangle\langle\Phi|$, where $|\Phi\rangle=\sum_{s}|s\rangle_{V} \otimes|s\rangle_{V^{\prime}} \in \mathcal{H} \otimes \mathcal{H}$ is an (unnormalized) maximally entangled state and $|s\rangle=\left|s_{1}, \ldots, s_{N}\right\rangle$ is an element of the computational basis, where $s_{n}=1, \ldots, d$. For a channel $\mathcal{E}$, its CJS is defined as $R=$ $\left(\mathcal{E}_{V} \otimes \mathbb{1}_{V^{\prime}}\right)(\Phi) \in L(\mathcal{H} \otimes \mathcal{H})$, where the identity channel acts on the ancillas. It fulfills $R=R^{\dagger} \geq 0$, and $\operatorname{tr}_{V}(R)=\mathbb{1}_{V^{\prime}}$. In fact, any $R$ satisfying these conditions defines a channel, whose action is then given by $\mathcal{E}(\rho)=\operatorname{tr}_{V^{\prime}}\left(\rho_{V^{\prime}}^{T} R\right)$, where the transpose is taken in the computational basis [28].

Given $A \in V$, we denote by $A^{\prime} \subset V^{\prime}$ the same set in the lattice of the ancillas. We can now characterize both CPQC and LPQC in terms of their CJS [29]:

Proposition 1. Given a channel $\mathcal{E}$, for all $A \in S$ there exist $\sigma_{A, \bar{A}^{\prime}}$ (and $\sigma_{B, \bar{B}^{\prime}}$ ) such that its CJS $R$ fulfills (i) $\operatorname{tr}_{a, \bar{B}}(R)=\sigma_{A, \bar{A}^{\prime}} \otimes \mathbb{1}_{\bar{B}^{\prime}}$ iff it is a CPQC; (ii) $\operatorname{tr}_{a, b}(R)=\sigma_{A, \bar{A}^{\prime}} \otimes \sigma_{B, \bar{B}^{\prime}}$ iff it is a LPQC.

The $\sigma$ 's are determined by the above equations, e.g., $\sigma_{A, \bar{A}^{\prime}}=\operatorname{tr}_{a, \bar{B}, \bar{B}^{\prime}}(R) / d^{\left|\bar{B}^{\prime}\right|}$. This proposition expresses that the CJS of CPQC and LPQC become decorrelated if we trace some of the qudits.

Tensor networks. - Let us now briefly recall the TN description of quantum states, operators, and channels $[22,23]$. Given a set of $N$ qudits in a graph $\mathcal{G}$, we associate with each vertex a tensor $A[n]$ with rank $z_{n}+1$, where $z_{n}$ is the coordination number of that vertex. We associate an index to each of the edges connecting that vertex and the other one to the corresponding qudit. The latter is called physical index and runs from $1, \ldots, d$, and the rest are the auxiliary indices, running from $1, \ldots, D$, the bond dimension. Then, we say that

$$
|\Psi\rangle=\sum_{s} c_{s}|s\rangle
$$

is a TN state of bond dimension $D$ if there exist tensors $A[n]$ of that bond dimension, such that each $c_{s}$ can be obtained by assigning the value $s_{n}$ to the physical index of $A[n]$ and contracting the rest of the indices according to the lattice [22,23]. For arbitrary lattices, they are called projected entangled pair states (PEPS). Analogously, TN can define operators and maps. For operators, we can replace $|s\rangle$ by $|s\rangle\left\langle s^{\prime}\right|$, so that now the tensors $B[n]$ have two physical indices each, and for maps the tensors $C[n]$ have four. They are called PEPO (or PEPU if they are unitary) and PEPM of bond dimension $D$, respectively. Any PEPU (PEPM) has the same TN description as the PEPS (PEPO) corresponding to its CJS and, thus, the same bond dimension. 
The graphical representation of TN [22,23] consists in replacing each tensor by a box, each index by a line, and contraction of indices by identifying the corresponding lines. For a graph $\mathcal{G}$, PEPS, PEPO, and PEPM are thus represented by the same graph where each of the vertices is replaced by a tensor that has one, two, and four lines with open ends, respectively, and otherwise they are connected according to the edges. We can block tensors to represent blocks of qudits. For instance, the representation of two PEPU, $U$ and $U^{\dagger}$, acting on sets $A a b B$ is shown in Figs. 1(b) and 1(c). We have written in each box the name of the set where the tensor acts and used an asterisk to specify that the tensor is transposed and complex conjugated. Figure 1(d) represents $U U^{\dagger}=\mathbb{1}$, where the multiplication is read from bottom to top. The bond dimension for the tensor corresponding to $A$ is $D^{z_{A}}$, where $z_{A}$ is the number of edges connecting $A$ with its neighborhood $a$, and the physical dimension is $d^{|A|}$. We can now define a notion that was introduced in Ref. [14].

Definition 3. We say that a PEPU is simple if for any $A \in S$

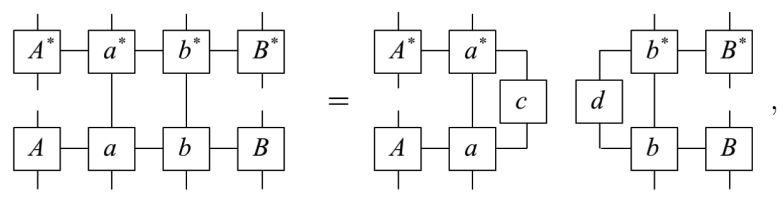

where

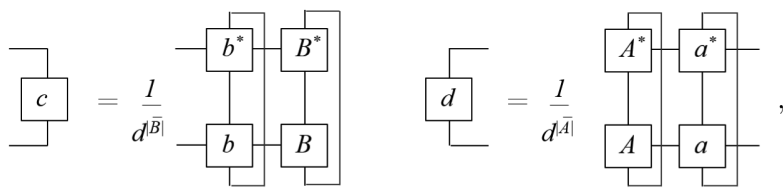

Quantum cellular automata.-We establish now the connection between QCA and PEPU as well as with LPQC.

Theorem 1. Given a unitary channel acting as $\mathcal{E}(\rho)=$ $U \rho U^{\dagger}$ on the qudits of a lattice, the following statements are equivalent: (i) $\mathcal{E}$ is a QCA (namely, a $\mathrm{CPQC}$ ). (ii) $\mathcal{E}$ is a LPQC. (iii) $U$ can be represented by a simple PEPU, where $D$ depends only on $d, d_{L}$, and $z$.

While all unitaries can be represented by PEPU, the last equivalence establishes that for a QCA this can be done efficiently, namely, with a bond dimension that does not depend on $N$. This has strong implications on the amount of entanglement that a unitary $U$ associated with a QCA can create between any two regions. If one applies $U$ to a pure product state $|\Psi\rangle$, then the entanglement of $U|\Psi\rangle$ between any set $A$ and the rest is $\leq D^{|\partial A|}$, where $|\partial A|$ is the number of edges between $A$ and $a$. This gives rise to an area law; to see that, we have to consider a sequence of QCA, $S_{E}=\left\{\mathcal{E}_{M}\right\}_{M=4}^{\infty}$, each acting on a lattice of $M^{d_{L}}$ qudits.
Furthermore, we denote by $E\left(A: A^{c}\right)$ the entanglement entropy [25] between the qudits in $A \subset V$ and its complement $A^{c}=V / A$ and by $\partial A$ their boundary.

Definition 4. A sequence of QCA obeys an area law if, for all $A \subset V$, the state obtained by applying any of the QCA to any pure product state fulfills $E\left(A: A^{c}\right) \leq c|\partial A|$, where $c$ is a constant independent of $M$.

Thus, Theorem 1 immediately implies that:

Corollary 1. Any sequence of QCA satisfies an area law.

General CPQC.-General CPQC possess very different properties than QCA. For instance, the set of CPQC acting on qudits in a lattice is convex. Note that this is not true for LPQC. Furthermore, whereas for QCA and any region $A \in S$,

$$
\mathcal{E}^{\dagger}\left(X_{A} Y_{B}\right)=\mathcal{E}^{\dagger}\left(X_{A}\right) \mathcal{E}^{\dagger}\left(Y_{B}\right)
$$

with $\mathcal{E}^{\dagger}\left(X_{A}\right)=X_{\bar{A}}, \mathcal{E}^{\dagger}\left(Y_{B}\right)=Y_{\bar{B}}$, this is not necessarily true for $\mathrm{CPQC}$.

Any channel (unitary or not) can be written in terms of a unitary operator through the Stinespring dilation [28]. In particular, we can consider the channel $\mathcal{E}$ built out of a QCA, $\mathcal{E}_{u}: L(\mathcal{H} \otimes \mathcal{H}) \rightarrow L(\mathcal{H} \otimes \mathcal{H})$, as

$$
\mathcal{E}(\rho)=\operatorname{tr}_{V^{\prime}}\left\{\mathcal{E}_{u}\left[\rho \otimes(|1\rangle\langle 1|)^{\otimes N}\right]\right\},
$$

where $|1\rangle$ is a state of the ancilla qudits [32]. Let us now introduce three other sets of channels:

Definition 5. We define $\mathrm{fQC}$ as the set of $\mathrm{CPQC}$ fulfilling the factorization condition (6), while tnQC is the set of CPQC whose CJS has a PEPO description (with bond dimension bounded by a function of $d, d_{L}$, and $z$ but not of $M$ ). Finally, we denote by dQC the set of CPQC that are obtained by a Stinespring dilation in terms of a QCA [that is, $\mathcal{E}_{u}$ in Eq. (7) is a QCA].

Let us give some illustrative examples. We take $d=2$, i.e., qubits, with $\left\{\left|s_{n}\right\rangle\right\}_{s_{n}=0}^{1}$ the local computational basis and $\sigma_{n}^{\alpha}$ the Pauli operators.

Example 1: A channel that is a tnQC but not a $\angle P Q C$.- - Let us define

$$
\mathcal{E}(\rho)=\frac{1}{2}\left[\rho+\left(\sigma^{x}\right)^{\otimes N} \rho\left(\sigma^{x}\right)^{\otimes N}\right] .
$$

$\mathcal{E}$ is a convex combination of two thQC with bond dimension $D=1$ and, thus, a tnQC with bond dimension $D=2$. Furthermore, it is also a CPQC, since it is a convex combination of two CPQC. However, it is not a LPQC, since it does not satisfy Proposition 1.

Example 2: A set of channels that are LPQC but not tnQC. - Let us consider the state (3), where $s_{n}=0,1$ and each qubit $n=\left(n_{1}, n_{2}, \ldots, n_{d_{L}}\right)$ is maximally entangled with the qubit $n^{\prime}=\left(n_{1}^{\prime}, n_{2}, \ldots, n_{d_{L}}\right)$, where $\left|n_{1}^{\prime}-n_{1}\right|=$ $M / 2$ with $M$ even. Let us define $R=\mathbb{1}_{V} \otimes \mathbb{1}_{V^{\prime}} / 2^{N}+S$, where 


$$
S=k_{N} \sum_{S} c_{s}\left[\otimes_{n=1}^{N}\left(\sigma_{n}^{x} \otimes \sigma_{n^{\prime}}^{x}\right)^{s_{n}}\left(\sigma_{n}^{z} \otimes \sigma_{n^{\prime}}^{z}\right)^{1-s_{n}}\right] .
$$

Choosing $k_{N}$ so that $\|S\|_{\infty} \leq 1 / 2^{N}$, we have $R \geq 0$, and tracing any system or ancilla qudit we get $\operatorname{tr}_{n}(S)=\operatorname{tr}_{n^{\prime}}(S)=0$. Thus, $\operatorname{tr}_{V}(R)=\mathbb{1}_{V^{\prime}}$ and, therefore, $R$ is a valid CJS that defines a channel $\mathcal{E}_{M}$ for each $M$. Furthermore, $R$ fulfills the conditions of Proposition 1, and it is therefore a LPQC. However, we claim that it does not admit a TN representation with finite bond dimension. Indeed, the latter is true iff $S$ can be represented as a PEPO with finite bond dimension. But the (unnormalized) state $|\Psi\rangle=\sum_{s} c_{s}|s\rangle$ is such that the rank of the reduced state in a hypercube of side $L<M / 2$ is $d^{\left(L^{\left.d_{L}\right)}\right.}$, so that its PEPS representation has a bond dimension that increases exponentially with $M$. But any PEPO representation of $S$ can be interpreted as PEPS for $|\Psi\rangle$ with the same bond dimension. We conclude that $R$ cannot be represented by a PEPO with bond dimension independent of $M$.

We are now in the position to formulate the following:

Theorem 2. For any of the considered lattices, $\mathrm{dQC} \subset \mathrm{fQC}=\mathrm{LPQC} \subset \mathrm{CPQC}$. Furthermore, $\mathrm{dQC} \subset \mathrm{tnQC}$ and $\operatorname{tnQC} \neq \mathrm{LPQC}$, where all inclusions are strict (see Fig. 2).

Note that this theorem does not say whether the intersection between tnQC and LPQC coincides with the set of dQC or simply contains it. This remains an interesting open question.

Finally, let us discuss an area law for the classes of QC defined above. As irreversible QC will typically create mixed states out of pure ones, rather than talking about the entanglement it is more appropriate to investigate the number of correlations that can be created. The relevant measure for this is the mutual information: Given a state $\rho$ in a qudit lattice, a subset of qudits $A \in V$, and its complement $A^{c}=V / A$, the mutual information is $I\left(A: A^{c}\right)=S_{A}+S_{A^{c}}-S_{V}$, where $S_{A}$ is the von Neumann entropy of the reduced state of the qudits in $A$ [25]. For Gibbs states of local Hamiltonians or for PEPO, it is known that the mutual information obeys an area law [33]. This motivates the following definition:

Definition 6. A sequence of QC obeys an area law if, for all $A \in V$, the state obtained by applying any of the QC to

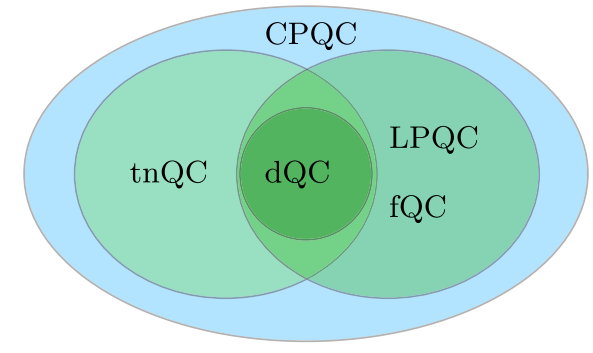

FIG. 2. Euler diagram for the class of channels defined in the main text, representing the statement of Theorem 2. any product state fulfills $I\left(A: A^{c}\right) \leq c|\partial A|$, where $c$ is a constant independent of $M$.

We can now state our third main result:

Theorem 3. Any sequence of LPQC obeys an area law.

Finally, we show that causality is not enough to bound the number of correlations that can be created when acting on a product state:

Example 3. Let us consider the dephasing channel acting on two qubits, $n$ and $m$ :

$$
\mathcal{E}_{n, m}(\rho)=\frac{1}{2}\left[\rho+\left(\sigma_{n}^{z} \otimes \sigma_{m}^{z}\right) \rho\left(\sigma_{n}^{z} \otimes \sigma_{m}^{z}\right)\right],
$$

and define the channel $\mathcal{E}=\bigotimes_{n \in V_{1}} \mathcal{E}_{n, n+e}$, where $V_{1}$ contains all $n \in V$ with $n_{1} \leq M / 2$ and $e=(M / 2,0,0, \ldots, 0)$. $\mathcal{E}$ is a convex combination of Pauli channels and, thus, a CPQC. However, the CJS is $R=\bigotimes_{n \in V_{1}} \rho_{n, n^{\prime}, n+e, n^{\prime}+e}$. The mutual information between $\left(n, n^{\prime}\right)$ and $\left(n+e, n^{\prime}+e\right)$ is one. Taking into account that the mutual information is additive under the tensor product, we conclude that, for a hypercube of side $L<M / 2$, it is $L^{d_{L}}$.

Conclusions. - We have investigated the connections between QCA, TN, and generation of quantum entanglement and correlations. We have shown that QCA can be efficiently represented by TN, implying an area law for the entanglement entropy that they generate. We have explored the implications of causality and locality for irreversible $\mathrm{QC}$, proving that only the former provides a constraint on the number of quantum correlations that can be created. Still, even LPQC cannot be represented efficiently via TN.

Our work opens up several questions and possibilities. The identification of QCA with PEPU allows one to use the established techniques based on TN for numerical simulations of their action $[34,35]$. This also gives us a very natural framework to investigate the classification of (symmetry-protected) topological (SPT) phases for QCA [18] in higher dimensions, with possible implications for the classification of Floquet SPT phases [13,36-38]. Additionally, QCA inherit the holographic principle of PEPS [39], which can also be used for their classification. Let us also mention some questions that our work immediately raises. Given that $\mathrm{CPQC}$ constitute a convex set, perhaps they can be obtained as the convex hull of either $\mathrm{dQC}$ or, more generally, LPQC. A solution to this problem would give us a very useful characterization of this set. In turn, this might be important in order to study equivalence classes of CPQC under smooth deformations.

We thank Alex Turzillo for discussions. L. P. acknowledges support from the Alexander von Humboldt foundation. J. I. C. acknowledges support by the EU Horizon 2020 program through the ERC Advanced Grant QENOCOBA No. 742102 and from the DFG (German Research Foundation) under Germany's Excellence StrategyEXC-2111-390814868. 
[1] T. Farrelly, arXiv:1904.13318.

[2] P. Arrighi, Nat. Comput. 18, 885 (2019).

[3] S. Richter and R. F. Werner, J. Stat. Phys. 82, 963 (1996).

[4] B. Schumacher and R. F. Werner, arXiv:quant-ph/0405174.

[5] P. Arrighi, V. Nesme, and R. Werner, in International Conference on Language and Automata Theory and Applications (Springer, New York, 2008), pp. 64-75.

[6] P. Arrighi, V. Nesme, and R. Werner, J. Comput. Syst. Sci. 77, 372 (2011).

[7] D. Gross, V. Nesme, H. Vogts, and R. F. Werner, Commun. Math. Phys. 310, 419 (2012).

[8] M. Freedman and M. B. Hastings, Commun. Math. Phys. 376, 1171 (2020).

[9] J. Haah, L. Fidkowski, and M. B. Hastings, arXiv:1812.01625.

[10] J. Haah, arXiv:1907.02075.

[11] M. Freedman, J. Haah, and M. B. Hastings, arXiv:1910.07998.

[12] X. Chen, Z.-C. Gu, and X.-G. Wen, Phys. Rev. B 84, 235128 (2011).

[13] H. C. Po, L. Fidkowski, T. Morimoto, A. C. Potter, and A. Vishwanath, Phys. Rev. X 6, 041070 (2016).

[14] J. I. Cirac, D. Perez-Garcia, N. Schuch, and F. Verstraete, J. Stat. Mech. (2017) 083105.

[15] M. B. Şahinoğlu, S. K. Shukla, F. Bi, and X. Chen, Phys. Rev. B 98, 245122 (2018).

[16] D. T. Stephen, H. P. Nautrup, J. Bermejo-Vega, J. Eisert, and R. Raussendorf, Quantum 3, 142 (2019).

[17] L. E. Hillberry, M. T. Jones, D. L. Vargas, P. Rall, N. Y. Halpern, N. Bao, S. Notarnicola, S. Montangero, and L. D. Carr, arXiv:2005.01763.

[18] Z. Gong, C. Sünderhauf, N. Schuch, and J. I. Cirac, Phys. Rev. Lett. 124, 100402 (2020).

[19] L. Fidkowski, H. C. Po, A. C. Potter, and A. Vishwanath, Phys. Rev. B 99, 085115 (2019).

[20] L. Piroli, A. Turzillo, S. K. Shukla, and J. I. Cirac, arXiv:2007.11905.

[21] G. K. Brennen and J. E. Williams, Phys. Rev. A 68, 042311 (2003).

[22] F. Verstraete, V. Murg, and J. I. Cirac, Adv. Phys. 57, 143 (2008).

[23] R. Orús, Ann. Phys. (Amsterdam) 349, 117 (2014).
[24] J. Eisert, M. Cramer, and M. B. Plenio, Rev. Mod. Phys. 82, 277 (2010).

[25] M. A. Nielsen and I. Chuang, Quantum Computation and Quantum Information (Cambridge University Press, Cambridge, England, 2002).

[26] For the lattices considered here, we can always block (i.e., merge into blocks [27]) $r^{d_{L}}$ qudits (assuming that $\tilde{M}=M / r \in \mathbb{N}$ ) and redefine the edges, so that the new lattice has $\tilde{N}=\tilde{M}^{d_{L}}$ qudits, and the range of a QCA with range $r$ becomes equal to one, although the coordination number may increase.

[27] J. I. Cirac, D. Perez-Garcia, N. Schuch, and F. Verstraete, Ann. Phys. (Amsterdam) 378, 100 (2017).

[28] M. M. Wolf, Lecture notes available at http://www-m5. ma. tum. de/foswiki/pub/M5 (2012).

[29] See Supplemental Material at http://link.aps.org/ supplemental/10.1103/PhysRevLett.125.190402 for a detailed proof of the statements presented in the main text, which includes Refs. [30,31].

[30] F. Verstraete, M. M. Wolf, D. Perez-Garcia, and J. I. Cirac, Phys. Rev. Lett. 96, 220601 (2006).

[31] H. Araki and E. Lieb, Commun. Math. Phys. 18, 160 (1970).

[32] In order to define the range of the QCA $\mathcal{E}_{u}$, one also needs to specify the sets of edges in the lattice made of the physical and ancillary vertices. Here we define it in the most natural way, by connecting each physical site with the corresponding ancilla.

[33] M. M. Wolf, F. Verstraete, M. B. Hastings, and J. I. Cirac, Phys. Rev. Lett. 100, 070502 (2008).

[34] U. Schollwöck, Ann. Phys. (Amsterdam) 326, 96 (2011).

[35] S.-J. Ran, E. Tirrito, C. Peng, X. Chen, L. Tagliacozzo, G. $\mathrm{Su}$, and M. Lewenstein, Tensor Network Contractions, Lecture Notes in Physics (Springer, New York, 2020).

[36] R. Roy and F. Harper, Phys. Rev. B 95, 195128 (2017).

[37] A. C. Potter and T. Morimoto, Phys. Rev. B 95, 155126 (2017).

[38] F. Harper, R. Roy, M. S. Rudner, and S. Sondhi, Annu. Rev. Condens. Matter Phys. 11, 345 (2020).

[39] J. I. Cirac, D. Poilblanc, N. Schuch, and F. Verstraete, Phys. Rev. B 83, 245134 (2011). 